\title{
Telomeres in disease
}

\section{Rodrigo Calado ${ }^{1}$ and Neal Young ${ }^{2 *}$}

\author{
Addresses: ${ }^{1}$ Department of Internal Medicine, University of São Paulo at Ribeirão Preto Medical School, Ribeirão Preto, SP, Brazil; ${ }^{2}$ Hematology \\ Branch, National Heart, Lung, and Blood Institute, National Institutes of Health, Bethesda, MD \\ *Corresponding author: Neal Young (youngns@nhlbi.nih.gov) \\ Fl000 Medicine Reports 2012, 4:8 (doi:10.34I0/M4-8) \\ This is an open-access article distributed under the terms of the Creative Commons Attribution-Non Commercial License \\ (http://creativecommons.org/licenses/by-nc/3.0/legalcode), which permits unrestricted use, distribution, and reproduction in any medium, \\ provided the original work is properly cited. You may not use this work for commercial purposes. \\ The electronic version of this article is the complete one and can be found at: http://f1000.com/reports/m/4/8
}

\begin{abstract}
Telomeres and telomere repair are basic molecular features of cells possessing linear DNA chromosomes and defects in them result in various diseases. This review examines recent advances in understanding these diseases, particularly at a molecular level, and in relating telomere dysfunction to clinical diseases. We also discuss the potential role of telomere elongation as a therapy in diseases, and more controversially, the prevention/reversal of aging.
\end{abstract}

\section{Introduction}

The ends of linear chromosomes have attracted serious scientific study, and Nobel Prizes, since the early 20th century. In the 1930s, Herman Muller and Barbara McClintock observed that fragmented chromosomes tended to fuse with each other yet normal chromosomes were stable, and they predicated characteristics of the "natural ends" or "telomeres" to explain this difference (Figure 1A). Almost 40 years later, Alexey Olovnikov, on theoretical grounds, and James Watson, based on phage experiments, recognized a fundamental problem in the semiconservative replication of DNA $[1,2]$. As DNA polymerase uses an RNA primer to start DNA synthesis, the space occupied by the RNA primers is not filled with newly synthesized DNA at the ends of chromosomes, so there would be inevitable loss of genetic information from the leading strand with every mitosis [3]. Elizabeth Blackburn, John Szostak, and Carol Greider in the 1980s solved this fundamental biologic problem by the discovery of the structure of telomeres, repetitive DNA elements at the ends of chromosomes, and the further elucidation of an active mechanism, whereby an enzyme repairs the loss of telomeres in proliferative cells (which they review in [4]).

Telomeres consist of hexameric nucleotide sequences that are repeated hundreds to thousands of times (TTAGGG in humans) at each extremity of the chromosome; the telomeric DNA is coated by a group of proteins, collectively termed shelterin proteins, which prevent the DNA repair machinery from mistaking telomeres for breaks in doublestranded DNA (Figure 1B) [5]. As telomeric sequences do not contain genes, they have the advantage that no genetic information is lost if they undergo erosion. At birth, white blood cells' telomeres are sufficiently long to allow about 200 cell divisions. With normal aging of an animal (and in cell culture), cells divide and telomeres shorten (Figure 1C). When telomeres become critically short, the nucleus signals the cell to cease proliferation, provoking cell senescence or cell death [6]. Telomere attrition explains the "Hayflick limit", the number of mitoses a cell is capable of undergoing in tissue culture before it stops dividing. Telomere length is therefore a type of "mitotic clock", a measure of a cell's proliferative history. Under circumstances in which cell proliferation continues despite critically short telomeres (usually about a few hundred hexanucleotides repeats on any chromosome), the telomere's protective function is lost. Subtelomeric genetic information can be lost and, more importantly, recombination between chromosomes occurs, leading to chromosome instability, aneuploidy, and transformation to a cancer phenotype.

Some proliferative cells can elongate telomeres enzymatically through the telomerase complex [7]. The enzyme telomerase (TERT) is a reverse transcriptase that employs a small RNA molecule (TERC) as a template to extend 
Figure I. Telomeres and telomerase

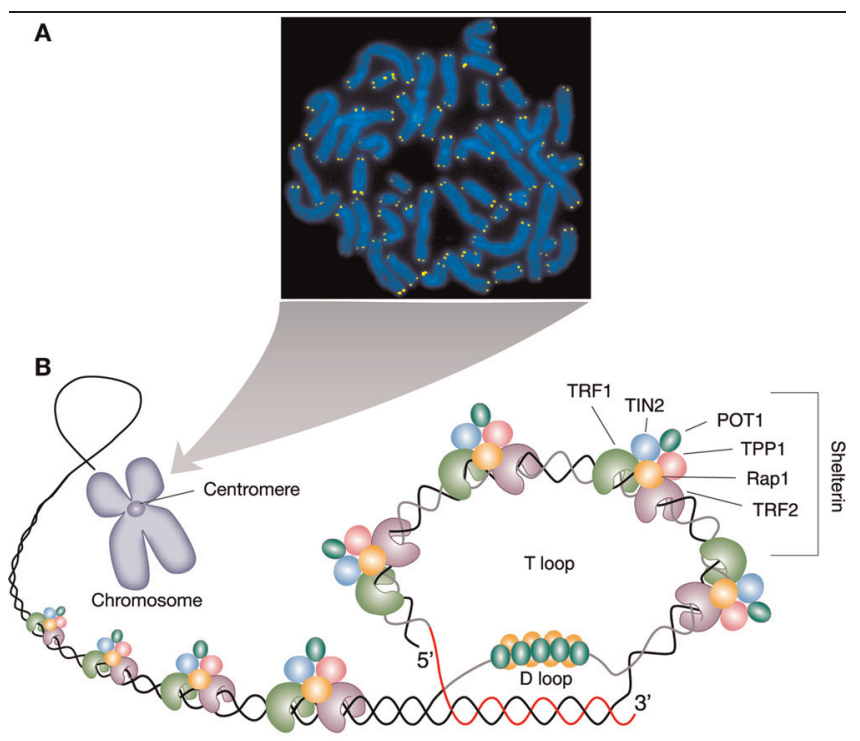

C

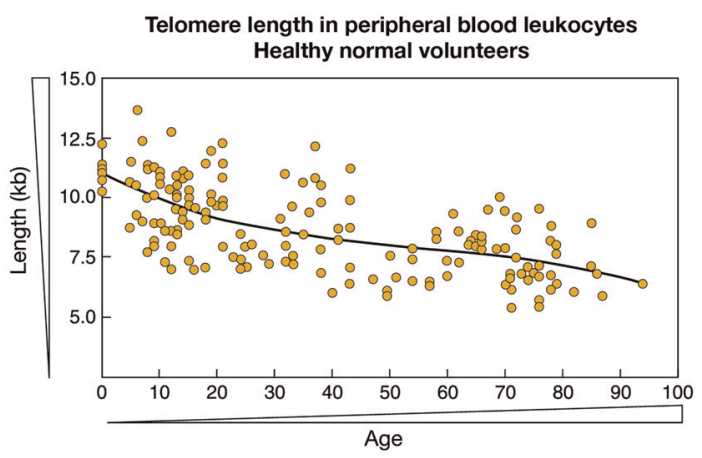

(A) Telomeres can be detected by fluorescent techniques (fluorescent in situ hybridization or FISH, yellow bright signals) at the tips of chromosomes in metaphase spreads (stained with DAPI). (B) This schematic representation of chromosomes and telomeres shows that telomeres are composed of thousands of tandem DNA repeat sequences. Protective proteins associated with telomere DNA are collectively termed shelterin or telosome (composed of TRFI, TRF2, TIN2, POTI, TPPI, and RapI). These proteins bind to telomeric DNA repetitive sequence, coating the nucleotide sequence. Additionally, the 3'overhang at the end of the leading strand falls back and anneals with the telomeric sequence, forming a lariat at the very end of the chromosome ( $T$ loop). The bottom panel $(C)$ illustrates the dynamics of telomere length as a function of aging in white blood cells. Telomeres shorten with aging, especially during the first two decades of life.

telomeres in cells. Thus, telomerase counterbalances the effects of cell division and cellular genetic "aging" in some populations, preventing senescence, cell death, and genetic instability. Telomere maintenance is also possible by other mechanisms. The alternative pathway (ALT) uses recombination between chromosomes; ALT is active in tumor cells, but its components and regulation are not as well understood [8].
Defects in telomeres result in various human diseases and recent years have seen big advances in our understanding of the causes at a molecular level.

\section{Telomere Diseases \\ Dyskeratosis congenita}

Telomere repair may be constitutionally defective due to mutations that severely reduce telomerase's capacity to elongate telomeres, leading to accelerated telomere attrition [9]. Dyskeratosis congenita, a rare inherited bone marrow failure disease, is the classic "telomere disease". The inherited defect in X-linked dyskeratosis congenita was identified in a gene named DKC1 by Inderjeet Dokal, in linkage studies of large pedigrees [10]. DKC1 encodes dyskerin, a protein that binds to the RNA component of telomerase and stabilizes the telomerase complex. Heterozygous mutations in TERC were later found in some families with autosomal dominant dyskeratosis congenita [11]. The severe phenotype of X-linked dyskeratosis congenita is likely due to hemizygous loss of DKC1 and markedly reduced telomerase function. In autosomal dominant dyskeratosis congenita, which results from defects in TERC and other autosomal chromosome genes, deficient telomerase function is caused by haploinsufficiency (where the level of the gene product is insufficient) of one gene.

Dyskeratosis congenita has pathognomonic features. Historically, the disease was first recognized by dermatologists, to whom patients still often present with abnormal nails and a pigmented reticular rash - oral leukoplakia completes this diagnostic triad. Aplastic anemia usually occurs in the first decade of life. Lungs and the liver are also affected in dyskeratosis congenita or fail after stem cell transplantation to correct the bone marrow disease.

\section{Telomeropathies in adults}

Dyskeratosis congenita with typical features can occasionally present late in life, such as in a patient with a DKC1 mutation whose blood cell counts do not recover after chemotherapy. However, most adult-onset telomeropathies result from mutations in TERC and in the enzymatic component encoded by TERT, which appear to behave more as genetic risk factors than as determinants for disease. The first TERT mutations found in humans were in adult patients with apparently acquired aplastic anemia, who lacked physical anomalies, and did not have a suggestive family history. Penetrance of the phenotype of TERT and TERC mutations is highly variable among and within such families, in severity, time of onset, and organ involved. Within pedigrees, members with the identical mutation may have no, or minimal, clinical manifestations, develop aplastic anemia late in life, or suffer 
pulmonary fibrosis or hepatic cirrhosis. Different organ systems may be affected in different family members at different times, and occasionally patients have disease in marrow, lung, and liver [9]. Telomerase mutations have been uncovered in a substantial minority of familial pulmonary fibrosis pedigrees, and in general populations of patients with severe cirrhosis.

Hematopoietic stem cells up-regulate telomerase in response to increases in daily demand for red blood cells, white blood cells, and platelets, so deficiencies in quantitative (stem cell number) and qualitative (repair) capacities would explain the bone marrow failure in the telomeropathies. However, it is harder to account for the problems telomeropathies cause in the liver and especially the lungs, as these are not usually considered organs with high replicative demand and the cell types affected are unknown. Conversely, the skin and mucous membranes are seldom affected in older patients, and strangely the gut is spared in both dyskeratosis congenita and other telomere diseases (absorptive function is maintained and patients do not develop mucositis), despite both tissues having high replication rates. To date, there are only anedoctal reports of anorectal or stomach adenocarcinomas. Similarly, many of the mechanisms of disease caused by telemeropathy are unknown. We don't know why fibrosis develops in the lungs and liver, while fat replaces marrow in aplastic anemia, or the contribution of the environment (immune attack on the marrow in aplastic anemia, smoking for the lungs, and alcohol and viral infection on the liver).

\section{Clinical implications}

The association of telomerase mutations with disease in three disparate organ systems (blood, lungs and liver) has important practical consequences for patients and their physicians. In the family history, the presence of even mild blood count abnormalities, pulmonary fibrosis, and hepatic cirrhosis are important clues to the diagnosis of a telomeropathy (as is acute myeloid leukemia - see below). Involvement of multiple medical subspecialties can be confusing for all concerned. One recent advance is that telomere length in leukocytes can now be measured commercially, and severe reduction of length is a reliable diagnostic marker [12]. TERT and TERC can also be sequenced. The finding of a telomerase deficit has consequences for prognosis, treatment, and genetic counseling. Although the diagnosis of telomeropathies can be straightforward, there may be complications. Some families with typical symptoms lack known mutations, and in others telomere length may be normal even in the presence of etiologic nucleotide substitutions. Rare mutations in shelterin genes can produce severe dyskeratosis congenita but do not alter telomerase repair capacity. Regulatory regions of genes, not routinely screened, may be responsible in some cases.

\section{Telomere shortening and cancer}

There are many sources of evidence that telomere attrition is associated with, and probably causative of, cancer. Patients with dyskeratosis congenita have a 1000fold increased risk of tongue cancer and about a 200-fold increased risk of acute myeloid leukemia [12]. In aplastic anemia, patients with the shortest telomeres (absent mutations) are 4-5-fold more likely to have their disease undergo malignant transformation to myelodysplasia and leukemia [13]. Telomere-free ends of chromosomes and aneuploidy are apparent in patients' bone marrows in tissue culture years before clinical symptoms. Furthermore, in acute myeloid leukemia without prior bone marrow failure, some patients have constitutional mutations in TERT and TERC [14].

Short leukocyte telomeres predict the development of cancer in Barrett's esophagus and in ulcerative colitis (which is associated with chromosome loss, gain, and rearrangement in cancer cells) $[15,16]$, but the mechanism is not clear. Do short telomeres of blood leukocytes mirror the telomere length of the affected organ, or are they a biomarker of exposure to reactive oxygen species in a chronic inflammatory process? Active oxygen can damage telomeres, and cells cultured in room air have excessive telomere shortening in comparison to cells cultured at low oxygen tension.

More generally, single nucleotide polymorphisms in TERT have been identified as risk factors in a multitude of genome-wide analyses in many cancers [17]. In a recent report, short leukocyte telomeres increased the risk of all cancers and of cancer fatalities in a large population followed over a decade [18]. Circumstantially, telomere attrition is an accompaniment of aging, itself the major risk for cancer (Figure 2). Iatrogenically, secondary hematologic malignancies occur after chemotherapy and radiation, which would be anticipated to lead to marrow stress and telomere shortening. More direct data come from a "knock-out" mouse model: crosses with reduced telomerase activity combined with diminished p53 surveillance developed a variety of epidermal cancers unusual in the rodent, but typical of humans.

In most malignancies, telomerase gene up-regulation or activation of the ALT pathway is thought necessary to establish cellular immortality. Telomerase is too frequently increased in cancer cells and cell lines to be considered an appropriate therapeutic target. Critically, short telomeres and a chromosome theory of the etiology of cancer are not in conflict with these findings. 
Figure 2. Telomere attrition and cancer risk

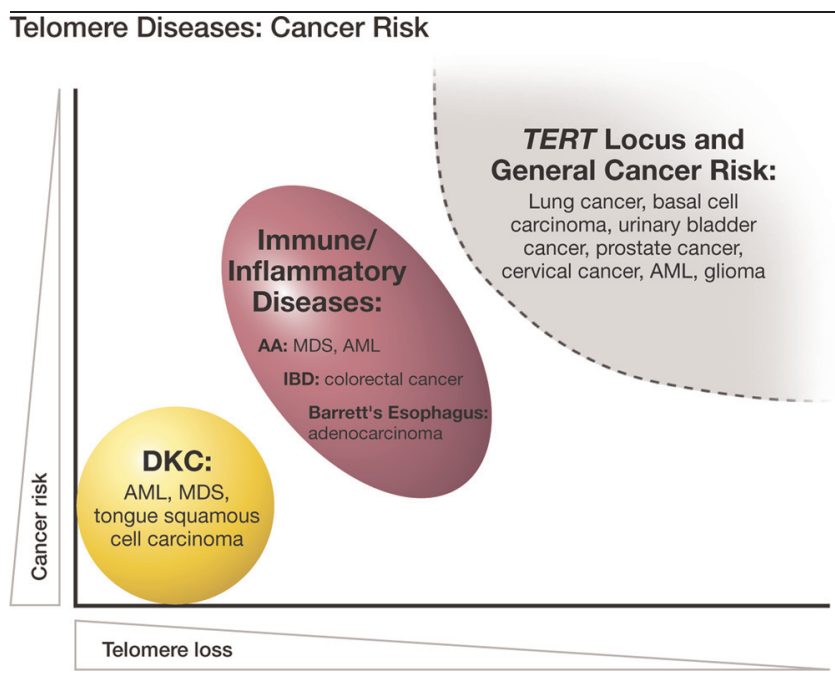

In dyskeratosis congenita (DKC), in which genetic penetrance is high, the risk for cancer development is also elevated, particularly for head and neck squamous cell carcinoma and acute myeloid leukemia (AML). Patients with aplastic anemia also are at risk of developing clonal malignant disorders, but at lower frequency. Similarly, short telomeres appear to predict progression of chronic inflammatory gastrointestinal states to cancer (aplastic anemia [AA] progresses to myelodysplastic syndrome [MDS] or AML, inflammatory bowel disease $[\mathrm{IBD}]$ progresses to colorectal cancers and Barrett's esophagus progresses to adenocarcinoma). In multiple genome-wide association studies, the TERT locus has appeared as a significant susceptibility locus for a variety of cancers, but at relatively low odds ratios.

Most cells in which telomere shortening reaches criticality likely either die or enter senescence. In those few that survive, perhaps due to inadequate monitoring by p53 and related DNA damage response safeguards, telomere repair would be subject to powerful selective pressure. Telomere shortening would also provide the equivalent of a "mutator phenotype", increasing spontaneous chromosomal aberrations, from numerical changes to structural abnormalities (translocations, insertions, deletions, and telomere associated end-to-end fusions), and therefore the pool of aberrant cells upon which selection would act.

\section{Telomeres and aging}

Telomeres shorten as we age. Consequently, telomere length has been postulated as a marker of "genetic age" (mitotic clock), as a fundamental explanation for the aging process, and has been marketed as a simple predictor of longevity. Telomere length assays have been bundled with recommendations for lifestyle modification and for drug therapy, neither based on appropriate clinical studies. Simple but appealing arguments relating telomeres and aging may be viewed, with some skepticism, as currently controversial, likely simplistic, and potentially harmful. Telomere length indeed reflects the cell's past proliferative history and future propensity to apoptosis, senescence, and transformation. However, cellular aging is not equivalent to organ or organismal aging.

There are several considerations in relating telomere biology to aging. First, physiologically there is overlap between the shortest telomere length of young children and the longest telomeres of the elderly. Most telomere shortening occurs early in life, in association with growth, and when the rate of disease in general is low. The paradigmatic telomere syndrome of dyskeratosis congenita is not at all typical of the progerias, inherited syndromes in which patients not only appear old but suffer diseases of aging, like premature atherosclerosis or dementia. Conversely, the organ damage of dyskeratosis congenita is not very similar to aging of marrow, lungs, and liver. The marrow becomes mildly hypocellular in older individuals but stem cell numbers may actually increase and blood counts remain stable; neither the liver nor lung normally become fibrotic with advanced age. Although relatively short telomeres of leukocytes have been associated with cardiovascular events among adults, the clinical correlations have not been consistent, and they may be related to overall reactive oxygen species exposure.

Studies in humans have attempted to relate short telomeres to longevity. In the provocative initial publication from the University of Utah, individuals around 60 years of age who had the longest telomeres lived longer than did subjects with the shortest telomeres, but the most associated cause of death in the latter group was, inexplicably, infection, and those with shorter telomeres did not have a higher rate of cancer deaths [19]. Heart disease as the cause of death was also more common in subjects with the shortest telomeres. Subsequent studies have produced conflicting findings. The Cardiovascular Health Study of subjects over 65 years of age found that individuals in the shortest quartile for telomere length were $60 \%$ more likely to die than those in the longest quartile [20]. Causes of death related to short telomeres were again infectious. Two twin studies at older age also correlated shorter telomeres with poorer survival $[21,22]$. Finally, an Italian cohort study that looked at participants at time zero and after 10 years found that death within 10 years was significantly more common in those with shorter telomeres [23].

In contrast, these associations have not been confirmed in other studies of older subjects. Blackburn and Cawthon reported that telomere length failed to predict survival, but correlated with years of healthy life [24]. 
In a Danish study of people aged 73 to 101 years, telomeres correlated with life expectancy in simple univariate analysis but, when corrected for age, did not predict longevity [25]. In Dutch men with a mean age of 78 years, telomere length eroded with aging but failed to correlate with mortality [26]. In a Finnish investigation, telomere length did not predict overall mortality [27]. Finally, in an analysis from California, short telomere length predicted death from cardiovascular disease in women but not in men, where the rate of shortening predicted mortality rather than length itself [28]. The discrepancies in these results may have several sources. In some analyses, telomere lengths may have been studied as a surrogate marker of age. In addition, retrospective studies may uncover "positive" associations that are random and not reproduced in follow-up investigations.

The telomere hypothesis of aging has also been tested in mice. For instance, in a murine model of telomerase deficiency and accelerated telomere attrition, researchers found that low telomerase expression deregulated certain intracellular pathways involved in mitochondrial function and glucose metabolism, ultimately causing heart muscle disease [29]. Interestingly, telomerase reactivation in these mice restored glucose production and heart function. However, the abnormalities observed in telomerase-deficient animals did not resemble those typical of humans with very short telomeres, in whom heart disease is rare. Translation of mouse experiments on telomeres to human physiology and disease should be approached with caution. Mice are not the ideal model for telomere attrition and its effects on aging: murine telomeres are 5 to 10 times longer than in humans, in spite of their much shorter lifespan. When telomerase is "knocked out" in mice, they live a healthy life for several generations; even though late generation animals with very short telomeres are infertile, they do not display the clinical phenotypes characteristic of human telomeropathies (bone marrow failure, pulmonary fibrosis, hepatic cirrhosis). Also, telomerase-deficient mice do not have a higher incidence of cancer, unless the p53 gene also is down modulated, in contrast to humans with telomerase deficiency, whom are at very high risk of developing cancer.

\section{Telomeres in regenerative medicine and telomerase modulation}

Telomeres and their repair are important in the new area of regenerative medicine. When Dolly the sheep was cloned from adult mammary gland cells more than a decade ago, her chromosomes were not exactly identical to the cells of the original sheep: Dolly's very short telomeres may have contributed to her illnesses, especially progressive lung disease [30]. In theory, this short-coming could be overcome by using embryonic stem cells, which express telomerase and are able to maintain their telomere lengths despite numerous cell divisions.

More recently, reprogramming of mature adult skin cells into the pluripotent state has been achieved with the introduction of just a few defined nuclear factors. During the process of reverting cells to a more immature state (with the pluripotency of embryonic stem cells), the reprogrammed cells' telomeres were highly elongated [31]. In the first steps of reprogramming, and likely in the early stages of embryogenesis, telomeres can be effectively elongated and cells can "rejuvenate" their telomeres. Since short telomeres limit cell proliferation, mechanisms that can potentially elongate telomeres are highly desirable tools for effective regenerative medicine.

Telomerase is expressed by a limited number of cell types, especially those with high proliferative capacity. However, even in these cells, telomerase expression is tightly regulated: just a few copies of the complex are present in the cell nucleus and it exerts its function during specific periods of the cell cycle. The mechanisms that modulate telomerase gene expression, resultant enzymatic activity, and affect telomere elongation are the focus of intensive research. MYC, a proto-oncogene that regulates the expression of many other genes and cell pluripotency, activates telomerase expression [32]. Sex hormones also activate telomerase expression in reproductive and non-reproductive organs, such as the bone marrow. The promoter region of the telomerase gene contains regulatory sequences that are modulated by estrogen; cells exposed to androgens (eventually converted into estrogens) or estrogens up-regulate telomerase expression [32]. In retrospect, the response of patients with aplastic anemia, especially children with inherited marrow failure, to androgens may be attributable to this mechanism. However, whether higher blood levels of sex hormones or exposure to exogenous sex hormones cause telomere elongation is still unknown.

\section{Conclusions}

Telomeres and telomere repair are basic molecular processes in cells possessing linear DNA chromosomes. It is clear that accelerated telomere attrition due to genetic defects in telomerase and in the gene encoding shelterin proteins is causative in several human diseases not previously thought to be related in the clinic: aplastic anemia, pulmonary fibrosis, and hepatic cirrhosis. The telomeropathies, especially in their milder and more chronic forms, may not be rare and are almost certainly often unrecognized by physicians. The potential for telomere repair in tissues under regenerative stress is of special interest, particularly given the reactive responses of 
fibrogenesis and adipogenesis and the role of telomere attrition in linking chronic inflammation to cancer in many organs and diseases. Yet we are still missing explanations for the genotype-phenotype anomalies seen in telomeropathies - highly variable telomerase gene penetrance, organ specificity, and clinical course. Both the genomic architecture modulating telomerase expression and the effect of the organism's environment on telomere attrition are poorly understood. Drugs or hormones that might modulate telomerase expression and maintain or elongate telomeres would be appealing in the treatment of the telomeropathies and in conditions in which telomere attrition has known medical consequences. Whether telomere shortening mediates human aging and whether telomere elongation may reverse aging or prevent agerelated diseases remain controversial.

\section{Competing interests}

The authors declare that they have no competing interests.

\section{References}

I. Olovnikov AM: Printsip marginotomii v matrichnom sinteze polinukleotidov. Dokl Akad Nauk SSSR I97I, 20I:I496-9.

FI000 Factor 10

Evaluated by Neal Young 15 Mar 2012

2. Watson JD: Origin of concatemeric T7 DNA. Nature New Biol 1972, 239:197-20|.

\section{FI000 Factor 6}

Evaluated by Neal Young 15 Mar 2012

3. Blackburn EH: Switching and signaling at the telomere. Cell 200I, 106:66I-73.

4. Blackburn EH, Greider CW, Szostak JW: Telomeres and telomerase: the path from maize, Tetrahymena and yeast to human cancer and aging. Nat Med 2006, I 2: I I33-8.

5. de Lange T: Shelterin: the protein complex that shapes and safeguards human telomeres. Genes Dev 2005, 19:2100-10.

6. Aubert G, Lansdorp PM: Telomeres and aging. Physiol Rev 2008, 88:557-79.

7. Greider $\mathrm{CW}$, Blackburn $\mathrm{EH}$ : Identification of a specific telomere terminal transferase activity in Tetrahymena extracts. Cell 1985, 43:405-13.

FI000 Factor 8

Evaluated by Neal Young 15 Mar 2012

8. Reddel RR: Alternative lengthening of telomeres, telomerase, and cancer. Cancer Lett 2003, 194:155-62.

FI000 Factor 6

Evaluated by Neal Young 15 Mar 2012

9. Calado RT, Young NS: Telomere diseases. N Engl J Med 2009, 36I:2353-65.

10. Heiss NS, Knight SW, Vulliamy TJ, Klauck SM, Wiemann S, Mason PJ, Poustka A, Dokal I: X-linked dyskeratosis congenita is caused by mutations in a highly conserved gene with putative nucleolar functions. Nat Genet 1998, 19:32-8.

FI000 Factor 6

Evaluated by Neal Young 15 Mar 2012

II. Vulliamy T, Marrone A, Goldman F, Dearlove A, Bessler M, Mason PJ, Dokal I: The RNA component of telomerase is mutated in autosomal dominant dyskeratosis congenita. Nature 2001, 413:432-5.

FI000 Factor 8

Evaluated by Titia De Lange 23 Oct 200 I

12. Alter BP, Baerlocher GM, Savage SA, Chanock SJ, Weksler BB, Willner JP, Peters JA, Giri N, Lansdorp PM: Very short telomere length by flow fluorescence in situ hybridization identifies patients with dyskeratosis congenita. Blood 2007, I I 0:|439-47.

FI000 Factor 6

Evaluated by Neal Young 15 Mar 2012

13. Scheinberg P, Cooper JN, Sloand EM, Wu CO, Calado RT, Young NS: Association of telomere length of peripheral blood leukocytes with hematopoietic relapse, malignant transformation, and survival in severe aplastic anemia. JAMA 2010, 304: I358-64.

FI000 Factor 10

Evaluated by Eva Guinan 27 Sept 201 I, Sharon Savage and Shahinaz Gadalla 9 Dec 2010, Albert N Békássy 9 Nov 2010

14. Calado RT, Regal JA, Hills M, Yewdell WT, Dalmazzo LF, Zago MA, Lansdorp PM, Hogge D, Chanock SJ, Estey EH, Falcão RP, Young NS: Constitutional hypomorphic telomerase mutations in patients with acute myeloid leukemia. Proc Natl Acad Sci U.S.A 2009, 106: I 187-92.

FI000 Factor 8

Evaluated by Laura Haneline 28 Apr 2009

15. O'Sullivan JN, Bronner MP, Brentnall TA, Finley IC, Shen W Emerson S, Emond MJ, Gollahon KA, Moskovitz AH, Crispin DA, Potter JD, Rabinovitch PS: Chromosomal instability in ulcerative colitis is related to telomere shortening. Nat Genet 2002, 32:280-4.

FI000 Factor 8

Evaluated by Neal Young 15 Mar 2012

16. Risques RA, Vaughan TL, Li X, Odze RD, Blount PL, Ayub K, Gallaher JL, Reid BJ, Rabinovitch PS: Leukocyte telomere length predicts cancer risk in Barrett's esophagus. Cancer Epidemiol Biomarkers Prev 2007, 16:2649-55.

FI000 Factor 8

Evaluated by Neal Young 15 Mar 2012

17. Rafnar T, Sulem P, Stacey SN, Geller F, Gudmundsson J, Sigurdsson A, Jakobsdottir M, Helgadottir H, Thorlacius S, Aben KKH, Blöndal T, Thorgeirsson TE, Thorleifsson G, Kristjansson K, Thorisdottir K, Ragnarsson R, Sigurgeirsson B, Skuladottir H, Gudbjartsson T, Isaksson HJ, Einarsson GV, Benediktsdottir KR, Agnarsson BA, Olafsson K, Salvarsdottir A, Bjarnason H, Asgeirsdottir M, Kristinsson KT, Matthiasdottir S, Sveinsdottir SG, et al.: Sequence variants at the TERT-CLPTMIL locus associate with many cancer types. Nat Genet 2009, 41:221-7.

FI000 Factor 8

Evaluated by Neal Young 15 Mar 2012

18. Willeit P, Willeit J, Mayr A, Weger S, Oberhollenzer F, Brandstätter A, Kronenberg F, Kiechl S: Telomere length and risk of incident cancer and cancer mortality. JAMA 2010, 304:69-75.

FI000 Factor 7

Evaluated by Lodovico Balducci 25 Aug 2010, Sharon Savage 21 Jul 2010

19. Cawthon RM, Smith KR, O'Brien E, Sivatchenko A, Kerber RA: Association between telomere length in blood and mortality in people aged 60 years or older. Lancet 2003, 36 I:393-5.

FI000 Factor 8

Evaluated by Neal Young 15 Mar 2012

20. Fitzpatrick AL, Kronmal RA, Kimura M, Gardner JP, Psaty BM, Jenny NS, Tracy RP, Hardikar S, Aviv A: Leukocyte telomere 
length and mortality in the Cardiovascular Health Study. J Gerontol A Biol Sci Med Sci 20I I, 66:42I-9.

FI000 Factor 6

Evaluated by Neal Young 15 Mar 2012

21. Kimura M, Hjelmborg JVB, Gardner JP, Bathum L, Brimacombe M, Lu X, Christiansen L, Vaupel JW, Aviv A, Christensen K: Telomere length and mortality: a study of leukocytes in elderly Danish twins. Am J Epidemiol 2008, 167:799-806.

FI000 Factor 6

Evaluated by Neal Young I5 Mar 2012

22. Bakaysa SL, Mucci LA, Slagboom PE, Boomsma DI, McClearn GE, Johansson B, Pedersen NL: Telomere length predicts survival independent of genetic influences. Aging Cell 2007, 6:769-74.

FI000 Factor 6

Evaluated by Neal Young 15 Mar 2012

23. Ehrlenbach S, Willeit P, Kiechl S, Willeit J, Reindl M, Schanda K, Kronenberg $F$, Brandstätter $A$ : Influences on the reduction of relative telomere length over 10 years in the populationbased Bruneck Study: introduction of a well-controlled highthroughput assay. Int J Epidemiol 2009, 38:1725-34.

FI000 Factor 6

Evaluated by Neal Young 15 Mar 2012

24. Njajou OT, Hsueh W, Blackburn EH, Newman AB, Wu S, Li R, Simonsick EM, Harris TM, Cummings SR, Cawthon RM: Association between telomere length, specific causes of death, and years of healthy life in health, aging, and body composition, a population-based cohort study. J Gerontol A Biol Sci Med Sci 2009, 64:860-4.

FI000 Factor 6

Evaluated by Neal Young 15 Mar 2012

25. Bischoff C, Petersen HC, Graakjaer J, Andersen-Ranberg K, Vaupel JW, Bohr VA, Kølvraa S, Christensen K: No association between telomere length and survival among the elderly and oldest old. Epidemiology 2006, 17:190-4.

26. Houben JMJ, Giltay EJ, Rius-Ottenheim N, Hageman GJ, Kromhout D: Telomere length and mortality in elderly men: the Zutphen Elderly Study. J Gerontol A Biol Sci Med Sci 20I I, 66:38-44.

FI000 Factor 6

Evaluated by Neal Young 15 Mar 2012
27. Strandberg TE, Saijonmaa O, Tilvis RS, Pitkälä KH, Strandberg AY, Miettinen TA, Fyhrquist F: Association of telomere length in older men with mortality and midlife body mass index and smoking. J Gerontol A Biol Sci Med Sci 20I I, 66:8I5-20.

FI000 Factor 6

Evaluated by Neal Young 15 Mar 2012

28. Epel ES, Merkin SS, Cawthon R, Blackburn EH, Adler NE, Pletcher MJ, Seeman TE: The rate of leukocyte telomere shortening predicts mortality from cardiovascular disease in elderly men. Aging 2009, I:8I-8.

FI000 Factor 6

Evaluated by Neal Young 15 Mar 2012

29. Sahin E, Colla S, Liesa M, Moslehi J, Müller FL, Guo M, Cooper M, Kotton D, Fabian AJ, Walkey C, Maser RS, Tonon G, Foerster F, Xiong R, Wang YA, Shukla SA, Jaskelioff M, Martin ES, Heffernan TP, Protopopov A, Ivanova E, Mahoney JE, Kost-Alimova M, Perry SR, Bronson R, Liao R, Mulligan R, Shirihai OS, Chin L, DePinho RA: Telomere dysfunction induces metabolic and mitochondrial compromise. Nature 2011, 470:359-65.

FI000 Factor 12

Evaluated by Lucio Comai 2I Mar 20II, Kevin Conley 16 March 2011

30. Shiels PG, Kind AJ, Campbell KH, Waddington D, Wilmut I, Colman A, Schnieke AE: Analysis of telomere lengths in cloned sheep. Nature 1999, 399:316-7.

FI000 Factor 6

Evaluated by Neal Young 15 Mar 2012

31. Marion RM, Strati K, Li H, Tejera A, Schoeftner S, Ortega S, Serrano M, Blasco MA: Telomeres acquire embryonic stem cell characteristics in induced pluripotent stem cells. Cell Stem Cell 2009, 4:| |4|-54.

FI000 Factor 8

Evaluated by Neal Young 15 Mar 2012

32. Calado RT, Yewdell WT, Wilkerson KL, Regal JA, Kajigaya S, Stratakis CA, Young NS: Sex hormones, acting on the TERT gene, increase telomerase activity in human primary hematopoietic cells. Blood 2009, I I 4:2236-43. 